\title{
Deep inferior epigastric perforator (DIEP) flap: Impact of drain free donor abdominal site on long term patient outcomes and duration of inpatient stay
}

Thacoor A, Torres-Grau J, Chana JS

\section{Background}

The deep inferior epigastric perforator (DIEP) flap is widely regarded as the Gold Standard in autologous breast reconstruction. There is currently a wide variation in use and duration of donor site abdominal drains and a paucity of evidence comparing outcomes and complications in patients with and without abdominal drains in DIEP reconstruction.

\section{Method}

A retrospective review of a database of patients who underwent DIEP breast reconstruction without abdominal drain insertion at the Royal Free Hospital between Jan 2012 and Nov 2016 was undertaken. Results were compared to previously published data from our group on patients undergoing DIEP breast reconstruction with abdominal drains between Jan 2011 and Jul 2012.

\section{Results}

A total of 35 patients underwent reconstruction with no abdominal drain (Group A). Of 74 patients who previously underwent reconstruction with abdominal drains, 41 patients underwent drain removal by postoperative day (POD) 3 regardless of output (Group B) and 33 underwent drain removal after POD 3 following instructions on drainage volume/24h (Group C). Total length of stay was shorter in Group A at 3.63 days vs 3.91 in Group B and 4.93 in Group C ( $=0.046, p<0.001$ ). There was no difference in total or specific complications between Group A vs Group B and Group C (11.43\% vs $12.12 \%$ and $21.95 \%, \mathrm{p}=0.93, \mathrm{p}=0.23$; seroma $(2.86 \%$ vs $0 \%$ and $4.88 \%, \mathrm{p}=0.31, \mathrm{p}=0.65)$, dehiscence $(8.57 \%$ vs $9.09 \%$ and $4.88 \%, \mathrm{p}=0.94, \mathrm{p}=0.52)$ and haematoma ( $0 \%$ vs $3.0 \%$ and $7.32 \%$, $\mathrm{p}=0.31, \mathrm{p}=0.10$ ).

\section{Discussion}

Our data suggests patients with no abdominal drains experience shorter inpatient hospital stays without increased postoperative complications.

\section{Conclusion}

We recommend against the use of donor site abdominal drains for DIEP reconstruction. 


\section{Introduction}

In 2016, 109256 women in the USA underwent breast reconstruction, with autologous flap reconstruction being performed in 20650 of them ${ }^{1}$. In the UK, the 2010 National Mastectomy and Breast Reconstruction Audit of 18216 women stated that free flaps were performed in 476 out of 3389 women who underwent immediate reconstruction and in 566 out of 1731 women who underwent delayed reconstruction ${ }^{2}$. The deep inferior epigastric perforator (DIEP) flap is fast becoming the Gold Standard in breast reconstruction due to its advantage of offering an autologous option replicating the feel of breast tissue as well as its associated lower donor site morbidity and overall better cost effectiveness ${ }^{34}$.

Complications associated with DIEP flap breast reconstruction are described in a 10-year retrospective review of 758 women; 5.9\% returned to theatre for flap-related issues, partial and total flap loss rates were $2.5 \%$ and $0.5 \%$ respectively, $12.9 \%$ developed fat necrosis, $5 \%$ developed abdominal donor site seromas and $0.7 \%$ developed postoperative abdominal herniae ${ }^{5}$. In addition, complication rates are reported to be even higher in bilateral DIEP flap reconstruction, with the risk of total flap loss being six times higher than in unilateral reconstruction ${ }^{6}$.

Donor site complications have been compared to those associated with elective abdominoplasty, due to the similar abdominal closure involved with lower rates of seroma in DIEP flap patients (3.5\% vs $16.1 \%$ ) and no differences in wound dehiscence ${ }^{7}$. Abdominal wall drains prevent fluid accumulation in the dead space resulting from tissue undermining and flap harvest, potentially minimising the above complication rates. However, they also represent a gateway for infection, are painful, limit patient mobility, require daily care upon discharge, potentially increase inpatient stay and ultimately resulting in an increased financial burden ${ }^{8}$.

There is currently minimal evidence to support both the use and duration of abdominal drains in DIEP flap reconstruction.

\section{Aim}

Data has been previously published by the Senior Author retrospectively comparing the length of inpatient stay and postoperative donor-site complications associated with early closed suction abdominal drain removal by postoperative day (POD) 3 irrespective of output, versus late removal (after POD3) based on drainage output and consistency in post-mastectomy DIEP flap reconstruction patients 9 .

The primary outcome of this study was to compare clinical outcomes associated with performing post-mastectomy DIEP flap reconstruction without the use abdominal drains (Group A) versus previously published data on early removal of drains by POD 3 (Group B) and late removal of drains after POD3 (Group C). 
Secondary outcome included analysis of duration of hospital inpatient stay between the above three groups.

\section{Methods}

A retrospective review of a prospectively collected electronic hospital patient database was performed to generate a spreadsheet of patients who underwent drain free donor site DIEP flap breast reconstruction by the Senior Author between January 2012 and November 2016, ensuring a minimum follow-up time of seven months for all patients.

Previously collected data by our group was analysed. This included a retrospective review of all patients who underwent unilateral DIEP flap breast reconstruction using donor site abdominal drains between January 2011 and July 2012, ensuring a minimum follow-up time of 1 year.

Patient notes were analysed for: age, date of birth, operation date, date of discharge, donor site complications including seroma, haematoma and wound dehiscence as well as flap-related or systemic complications. Complications were defined as per previous publication ${ }^{9}$.

All data were presented as mean \pm s.d.. The difference in patient demography between the three groups were compared using one- way ANOVA followed by Bonferroni test for multiple comparisons. Difference in complication between groups were compared using Fisher's exact test. A $\mathrm{p}$ value of less than 0.05 was considered significant. All analysis were conducted using SPSS version 22 (IBM,Armonk, NY, USA).

\section{Surgical technique}

The DIEP flap harvest was performed in a standard fashion. A layer of fat deep to Scarpa's fascia was preserved over the anterior rectus sheath to preserve cutaneous lymphatic collectors of the abdominal wall. Donor-site closure was performed first by quilting Scarpa's fascia to the anterior rectus sheath using 3/0 PDS sutures to obliterate any dead space. The wound edges were then approximated using 3/0 monocryl sutures through Scarpa's fascia followed by layered closure using 3/0 monocryl deep dermal sutures and a running subcuticular 3/0 prolene skin suture. Additionally, two size 16-French Redivac abdominal drains were placed in patients in Groups B and C only.

\section{Results}

One hundred and nine women underwent unilateral DIEP flap breast reconstruction between January 2011 and November 2016 (35 Group A, 41 Group B, 33 Group C) with a minimum follow-up time of seven months. Patient demography is summarised in Table 1. No patient was discharged home with abdominal drains, where used. 


\begin{tabular}{|c|c|c|c|c|}
\hline & Group A & Group B & Group C & p value \\
\hline Age & $54.7 \pm 10.5$ & $54.1 \pm 7.0$ & $50.9 \pm 10.3$ & 0.175 \\
\hline BMI & $28.7 \pm 2.16$ & $29.11 \pm 2.46$ & $28.96 \pm 2.63$ & 0.782 \\
\hline Smoking & 2 & 0 & 1 & 0.509 \\
\hline $\begin{array}{c}\text { Neo-adjuvant } \\
\text { chemotherapy }\end{array}$ & 28 & 24 & 30 & 0.729 \\
\hline Previous surgery & 18 & 20 & 20 & 0.529 \\
\hline Radiotherapy & 26 & 21 & 29 & 0.624 \\
\hline & $3.6 \pm 0.6$ & $3.9 \pm 0.4$ & $4.9 \pm 0.8$ & $\begin{array}{c}\text { B p }=0.204 . \\
\text { Group C vs } \\
\text { B or A } \\
\text { p=0.001 }\end{array}$ \\
\hline Length of stay & & & &
\end{tabular}

Table 1: Patient demography

There is no significant difference in the length of stay between patients in Group A and B (3.6 \pm 0.6 vs $3.9 \pm 0.4 ; p=0.204)$.. Length of stay of patient in Group $C$ is significantly higher compared to Group A ( $\mathrm{p}=0.001)$ and Group B ( $\mathrm{p}=0.001)$.

There were no statistically significant differences in total ( $11.43 \%$ vs $12.12 \%$ vs $17.07 \%, \mathrm{p}=0.780$ ) or specific postoperative complications between patients in the three groups (Table 1). The overall relative risk of no drains was 0.67 compared to late removal of drains, while it was 0.71 for early removal of drains compared to late removal of drains. Rates of seroma were $2.86 \%$ vs $0 \%$ vs $4.88 \%$ $(\mathrm{p}=0.774)$; rates of wound dehiscence were $8.57 \%$ vs $9.09 \%$ vs $4.88 \%$ ( $\mathrm{p}=0.728)$; rates of haematoma were $0 \%$ vs $3.00 \%$ vs $7.32 \%$ ( $\mathrm{p}=0.0 .316$ ) between Groups A, B and C respectively.

\begin{tabular}{|l|l|l|l|l|}
\hline & Total \% & Seroma \% & Dehiscence \% & Haematoma \% \\
\hline Group A & 11.43 & 2.86 & 8.57 & 0.00 \\
\hline
\end{tabular}




\begin{tabular}{|l|l|l|l|l|}
\hline Group B & 12.12 & 0.00 & 9.09 & 3.00 \\
\hline Group C & 17.07 & 4.88 & 4.88 & 7.32 \\
\hline P values & 0.780 & 0.774 & 0.728 & 0.316 \\
\hline & & & & \\
\hline
\end{tabular}

Table 1. Total and specific complication rates between the 3 groups

\section{Discussion}

There is a growing debate in the literature regarding DIEP flap breast reconstruction, relating to both the use and the duration of donor site abdominal drains. In patients where abdominal drains are used, our group has previously published evidence-based recommendations on the timing of drain removal in DIEP, transverse rectus abdominis myocutaneous (TRAM) and latissimus dorsi (LD) flap breast reconstruction, advocating early removal by POD3 ${ }^{91011}$. However, although several reports have also been published on obviating the need for drain use altogether during abdominal wall closure, the majority of them relate to abdominoplasty with minimal data on the implications for perforator based flap reconstruction.

This study has demonstrated that performing a drain free donor site DIEP flap breast reconstruction by using quilting sutures and preserving the deep-layer abdominal fat was associated with a shorter inpatient hospital stay as well as similar rates of postoperative complications to cases where abdominal drains had been used. This could imply safely avoiding abdominal drains as part of DIEP flap breast reconstruction, thus also limiting their associated drawbacks. Drains are painful, limit patient mobility and create a potential entry site for infections.

The evidence for quilting sutures reducing complication rates in DIEP breast reconstruction is controversial. Liang et $\mathrm{al}^{12}$ performed a comparative study of abdominal closure using progressive tensioning with a running barbed suture quilting technique in breast reconstruction with the DIEP flap and reported a reduction in abdominal drainage. Similar results were reported by Nagarkar et $\mathrm{al}^{8}$ where the use of running barbed progressive tension sutures only was not associated with increased complication rates versus closure with interrupted progressive tension sutures with abdominal drain placement versus closure with abdominal drains only. Quilting sutures have also been shown to be of benefit reducing incidence of donor site seroma formation in TRAM flap breast reconstruction ${ }^{13}$. However, contrasting results were reported by McCarthy et $\mathrm{al}^{14}$, where quilting sutures resulted in no significant decrease in seroma formation. We have demonstrated that our reported rates of seroma in patients with no abdominal drains were lower than those reported in the literature (2.8\% vs $3.5 \%)^{7}$.

Quilting by progressive tension suturing was first described by Pollock and Pollock ${ }^{15}$ in abdominoplasty as a way of partially transferring wound tension in the flap to the fascial system and 
evenly distributing tension over the full length of the flap, allowing tension-free wound closure. This could explain why quilting in our study could also be a valuable adjunct at reducing rates of wound dehiscence.

Our surgical technique used involved the preservation of fat tissues deeper to Scarpa's fascia overlying the anterior rectus sheath. Evidence for this practice in DIEP flap harvesting has not been reported in the literature, although conflicting evidence exists for its use in TRAM harvesting and abdominoplasty. Nagasao et $\mathrm{al}^{16}$ noted that preservation of deep-fat tissues in zone 3 and 4 in TRAM flap harvest reduced postoperative donor site fluid exudates which enabled earlier removal of drains. This further supports results from a morphometric study by Costa-Ferreira recommending a more superficial plane of dissection in the infraumbilical area during abdominoplasty ${ }^{17}$. However, conflicting results were published by Tourani et a ${ }^{18}$ following the superficial lymphatic drainage of eight hemiabdomen specimens from four human cadavers.

There are several limitations to this study. Firstly, it is largely limited by its retrospective nature, small sample sizes and short minimum follow-up times, particularly in Group A. Certain factors potentially impacting on postoperative outcomes, including BMI, smoking status and adjuvant chemotherapy were not analysed as part of this study. The quality of abdominal closure could also have been inconstant, as it was often performed by varying members of the surgical team, including senior house officers, specialist registrars and senior surgeon, each with varying surgical skill. Furthermore, this study examines a single-surgeon technique, which could lead to variable results when reproduced by others. We thus welcome larger prospective, international, randomized controlled trials to further support our findings.

\section{Conclusion}

Our study has shown that a donor site drain free DIEP flap breast reconstruction leads to a shorter inpatient hospital stay with no statistically significant increase in complication rates. Benefits for the patient would include less postoperative pain, potentially reduced risk of drain-related infection and improved mobility with less time spent in hospital. This would support the principles of enhanced recovery and fast-track surgery as mobility would be enhanced without hindrance previously resulting from abdominal drains. Furthermore, it would concomitantly result in financial savings for hospital from shorter inpatient stay and reduced drain-related management outpatient visits. 


\title{
References
}

\begin{abstract}
${ }^{1}$ American Society of Plastic Surgeons. American society of plastic surgeons report of the 2016. Plastic surgery statistics. 2016

2 The NHS. information centre. National mastectomy and breast reconstruction audit 2010. http://www.rcseng.ac.uk/ surgeons/research/surgical-research/docs/nationalmastectomy-and-breastreconstruction-audit-third-report- 2010
\end{abstract}

${ }^{3}$ Knox AD, Ho AL, Leung L, Tashakkor AY, Lennox PA, Van Laeken N, Macadam SA. Comparison of Outcomes following Autologous Breast Reconstruction Using the DIEP and Pedicled TRAM Flaps: A 12-Year Clinical Retrospective Study and Literature Review. Plast Reconstr Surg. 2016 Jul;138(1):16-28

${ }^{4}$ Krishnan NM, Purnell C, Nahabedian MY, Freed GL, Nigriny JF, Rosen JM, Rosson GD. The cost effectiveness of the DIEP flap relative to the muscle-sparing TRAM flap in postmastectomy breast reconstruction. Plast Reconstr Surg. 2015 Apr;135(4):948-58

${ }^{5}$ Gill PS, Hunt JP, Guerra AB, Dellacroce FJ, Sullivan SK, Boraski J, Metzinger SE, Dupin CL, Allen RJ. A 10year retrospective review of 758 DIEP flaps for breast reconstruction. Plast Reconstr Surg. 2004 Apr $1 ; 113(4): 1153-60$

${ }^{6}$ Wade RG, Razzano S, Sassoon EM, Haywood RM, Ali RS, Figus A. Complications in DIEP Flap Breast Reconstruction After Mastectomy for Breast Cancer: A Prospective Cohort Study Comparing Unilateral Versus Bilateral Reconstructions. Ann Surg Oncol. 2017 Jun;24(6):1465-1474

${ }^{7}$ Salgarello M, Tambasco D, Farallo E. DIEP flap donor site versus elective abdominoplasty short-term complication rates: a meta-analysis. Aesthetic Plast Surg. 2012 Apr;36(2):363-9

${ }^{8}$ Nagarkar P, Lakhiani C, Cheng A, Lee M, Teotia S, Saint-Cyr M. No-drain DIEP Flap Donor-site Closure Using Barbed Progressive Tension Sutures. Plastic and Reconstructive Surgery Global Open. 2016;4(4):e672

${ }^{9}$ Miranda BH, Amin K, Chana JS. The drain game: abdominal drains for deep inferior epigastric perforator breast reconstruction. J Plast Reconstr Aesthet Surg. 2014 Jul;67(7):946-50

${ }^{1010}$ Miranda BH, Wilson RB, Amin K, Chana JS. The drain game: Abdominal drains for transverse rectus abdominis myocutaneous breast reconstruction. J Plast Reconstr Aesthet Surg. 2015 Jun;68(6):810-4

${ }^{11}$ Miranda BH, Amin K, Chana JS. The drain game: back drains for latissimus dorsi breast reconstruction. J Plast Reconstr Aesthet Surg. 2014 Feb;67(2):226-30

${ }^{12}$ Liang DG, Dusseldorp JR, van Schalkwyk C, Hariswamy S, Wood S, Rose V, Moradi P. Running barbed suture quilting reduces abdominal drainage in perforator-based breast reconstruction. J Plast Reconstr Aesthet Surg. 2016 Jan;69(1):42-7

${ }^{13}$ Rossetto LA, Garcia EB, Abla LE, Ferreira LM. Seroma and quilting suture at the donor site of the TRAM flap in breast reconstruction: a prospective randomized double-blind clinical trial. Ann Plast Surg. 2014 Apr;72(4):391-7

${ }^{14}$ McCarthy C, Lennox P, Germann E, Clugston P. Use of abdominal quilting sutures for seroma prevention in TRAM flap reconstruction: a prospective, controlled trial. Ann Plast Surg. 2005 Apr;54(4):361-4

${ }^{15}$ Progressive tension sutures: a technique to reduce local complications in abdominoplasty. Pollock H, Pollock T Plast Reconstr Surg. 2000 Jun; 105(7):2583-6; discussion 2587-8 
${ }^{16}$ Nagasao T, Tamai M, Moromomi T, Miki T, Kogure T, Hamamoto Y, Kudo H, Tanaka Y. Preservation of deep-layer fat of lateral zones prevents postoperative seroma after TRAM-flap harvesting for breast reconstruction: a retrospective study. J Plast Surg Hand Surg. 2017 Jan 13:1-9

${ }^{17}$ Costa-Ferreira A, Marco R, Vásconez L, Amarante J. Abdominoplasty With Scarpa Fascia Preservation. Ann Plast Surg. 2016 Jun;76 Suppl 4:S264-74

${ }^{18}$ Tourani SS, Taylor GI, Ashton MW. Scarpa Fascia Preservation in Abdominoplasty: Does It Preserve the Lymphatics? Plast Reconstr Surg. 2015 Aug;136(2):258-62 